Revista científica, arbitrada e indizada, bajo la modalidad electrónica.

Recibido: 22/06/2019

Aceptado: 15/07/2019

\title{
Futuro y prospección de la innovación en empresas del sector logístico
}

Future and prospecting of innovation in companies in the logistics sector

\author{
Yazmín Dorati \\ ydcantu@ultina.edu.pa \\ Instituto de Logística y Cadena de Suministro. \\ Universidad Latina de Panamá. \\ Humberto Álvarez \\ humberto.alvarez@utp.ac \\ Universidad Tecnológica de Panamá.
}

\begin{abstract}
RESUMEN
Los vertiginosos cambios en la tecnología aunado a la transformación constante de los gustos de los consumidores contribuyen a la imperiosa necesidad de innovar. Las empresas logísticas son las encargadas de la disponibilidad de los productos y servicios y en el proceso deben crear valor mediante la innovación, a fin de no quedar rezagadas. El presente análisis es un estudio proyectivo a fin de colocar en un alto nivel de innovación en un futuro a empresas logísticas. En esa virtud, mediante la metodología prospectiva y el Método Delphi, se detectaron las falencias del sistema, también muestra los diferentes escenarios que pueden suscitarse para que las empresas en el ámbito logístico obtengan un posicionamiento sólido en materia de innovación. Los instrumentos de la metodología prospectiva fueron: Encuesta aplicada a 73 empresas del sector y/o que están íntimamente ligadas. Así mismo, el análisis documental y el Método Delphi de expertos para las evaluaciones. Para este último se entrevistaron a 13 expertos en la materia quienes aportaron la información inicial. Otros elementos de análisis fueron la MIC MAC (matriz de impactos cruzados, Técnica Mactor, Técnica Delphi, Técnica SMIC y los Métodos de Escenarios y para el análisis estadístico se utilizó SPSS y Excel. Los resultados identificaron 3 elementos: la falta de un centro de innovación en logística y cadena de suministro que sirva de laboratorio para gestar novedades; carencia de gestión creativa e innovación dentro de las empresas y, por último, el monitoreo del mercado para conocer los cambios en las preferencias de los consumidores en tiempo real. Para el adecuado desarrollo de la innovación deben intervenir ciertos actores: El gobierno, clientes, centros de investigación y desarrollo para trasferir tecnología, centros educativos, mercado, la competencia, la empresa con características innovadoras, Senacyt, y Asociaciones Gremiales.
\end{abstract}

Palabras clave: Innovación, prospectiva, empresas logísticas 


\section{Revista científica, arbitrada e indizada, bajo la modalidad electrónica.}

\section{ABSTRACT}

The vertiginous changes in technology coupled with the constant transformation of consumer tastes contribute to the imperative need to innovate. Logistics companies are responsible for the availability of products and services and in the process must create value through innovation, so as not to lag behind. This analysis is a projective study in order to place logistics companies in a high level of innovation in the future. In that virtue, by means of the Prospective Methodology and the Delphi Method, the flaws of the system were detected, it also shows the different scenarios that can arise for companies in the logistics field to obtain a solid positioning in terms of innovation. The instruments of the prospective methodology were: Survey applied to 73 companies in the sector and / or that are closely linked. Likewise, the documentary analysis and the Delphi Method of experts for evaluations. For the latter, 13 experts in the field were interviewed who provided the initial information. Other elements of analysis were the MIC MAC (Cross Impact Matrix, Mactor Technique, Delphi Technique, SMIC Technique and Scenario Methods and for statistical analysis SPSS and Excel were used. The results identified 3 elements: the lack of an Innovation Center in Logistics and Supply Chain that serves as a laboratory to manage new developments; lack of creative management and innovation within companies and, finally, market monitoring to learn about changes in consumer preferences in real time. For the proper development of innovation, certain actors must intervene: The government, customers, Research and Development Centers to transfer technology, Educational Centers, market, competition, the company with innovative features, Senacyt, and Trade Associations.

Key words: Innovation, prospective, logistics companies

\section{INTRODUCCIÓN}

Planificar en un ambiente tan competitivo es muy complejo. Las culturas latinoamericanas no están acostumbradas a la programación, mucho menos a prever situaciones inesperadas que les permita preparar planes de contingencia con la antelación que se debe para dirigir los resultados hacia los objetivos trazados por la empresa.

Muchas de las causas se deben a que las empresas se hallan inmersas en la operatividad y la cotidianeidad caracterizada por la presión de la urgencia por complacer a un mercado cada día más exigente.

Para romper con ese esquema se deben realizar ejercicios sencillos en donde se cuestione ¿Cuál es la posición en aspectos de innovación que desea tener la empresa en un futuro? ¿Cuáles son los actores que deben intervenir para cumplir cabalmente con esas metas? ¿Cuáles son los escenarios posibles para que se cumplan los objetivos?

La razón por la cual se seleccionaron empresas del área logística se debe a que es un sector de alto impacto en la economía de cualquier parte del mundo, cuya función de producir bienes y servicios, abastecer el mercado, garantiza la disponibilidad de mercancías para la sustentación de la vida misma.

Por lo tanto, un estudio prospectivo permitirá establecer el posicionamiento futuro del sector que se desea tener y quiénes son los actores que deben intervenir a fin de dar cabal 


\section{Revista científica, arbitrada e indizada, bajo la modalidad electrónica.}

cumplimiento a las metas programadas. Con esos datos se realizan los ajustes necesarios en el presente, con el único fin de adecuar estrategias y tácticas amoldándolas a la meta futura. Las técnicas de recolección de datos son las distintas formas o maneras de obtener la información. En este caso: la entrevista a expertos, la encuesta y el análisis documental. Para el análisis estadístico: Statistical Package for the Social Sciences (SPSS).

\section{DESARROLLO}

\section{La prospectiva y la innovación}

El término prospectiva fue creado por Gastón Berger en contraposición a retrospectiva (Instituto de Prospectiva Estratègica, IPE, 1995), definía la prospectiva como "la ciencia que estudia el futuro para comprenderlo y poder influir en él" (Inayatullah, 2012). Es decir, se trata de que estudiando e imaginando el futuro (Inayutallah, 1990), se establezcan las bases para transformar el presente (Moctezuma \& Tapia, 2012) (Mojica, 1991). De esta forma, "el futuro es la razón de ser del presente". Berger añadía y recalcaba que mediante la prospectiva se debía "observar lejos, ampliamente y profundamente, pensar en el hombre y asumir riesgos (Montañana, 2004).

Michel Godet fue el creador de varias herramientas estratégicas (Pinto, 2008), de prospectiva desarrolladas a mediados de los setenta (Godet M. , 2000), sin embargo, el precedente que se tiene de quien impulsó por primera vez dicho enfoque fue Jay Forrester en 1961 (Forrester, 1961); en 1974 Godet y Duperrin, buscaron operacionalizar dicho método en un primer estudio sobre la energía nuclear en Francia.

De acuerdo con el Instituto de Prospectiva Estratégica de Godet (Guzmán, 2005), la prospectiva se aplica para explorar las posibles y/o probables evoluciones futuras de empresas, grupos, sectores, organizaciones, instituciones, temáticas o problemáticas de toda índole (políticas, económicas, tecnológicas, sociológicas, etc.) a medio y largo plazo, mediante el análisis de las variables que más influirán en la evolución y teniendo en cuenta los comportamientos de los actores implicados (Baena, 2005).

De acuerdo a (Godet \& Durance, 2011), la Prospectiva se compone del Análisis Estructural o Método MICMAC (Matriz de Impactos Cruzados, Técnica Mactor, Técnica Delphi, Técnica SMIC y los Métodos de Escenarios (Ancelin, Nov 1983.)

Al respecto, la Matriz de Impactos Cruzados Multiplicación Aplicada para una clasificación (MICMAC) o matriz de análisis estructural (Tabla 1): El análisis estructural es una herramienta de estructuración de una reflexión colectiva (Godet M. \&., 2011). Ofrece la posibilidad de describir un sistema con ayuda de una matriz que relaciona todos sus elementos constitutivos. Partiendo de esta revisión, este método tiene por objetivo, hacer aparecer las variables influyente (o motrices) y dependientes, clasificándolas en directas, indirectas, o potencial, y por ello, las variables esenciales a la evolución del sistema (prospective, s.f.), (Saint Paul R., 1974).

El índice de motricidad significa las veces que la variable influye en las restantes. No significa que las variables se clasifican como influyentes, ya que eso dependerá en donde su ubique en el diagrama de dispersión. (Estrada Ruiz, 2014). 


\section{Revista científica, arbitrada e indizada, bajo la modalidad electrónica.}

El índice de dependencia significa las veces que la variable es influida por las restantes. No significa que las variables se clasifican como dependientes, ya que eso dependerá en donde su ubique en el diagrama de dispersión

El método Delphi (Chitu \& Suzanne, 2004), es un método práctico que es utilizado para la resolución y determinación de problemas de forma abierta; es una técnica de investigación que se utiliza con el propósito de realizar pronósticos y predicciones (Conceptodefinición.com, s.f.). Este método busca la convergencia de opiniones de los expertos en un tema en específico utilizando cuestionarios (Miklos, 1995). En este estudio la colaboración de estos expertos dio como resultado las evaluaciones en las siguientes herramientas: Mic-Mac (Tabla 1), Matriz de actores (Tabla 3) y Matriz de impactos cruzados (Tabla 4).

La Técnica Smic: Sistema y matrices de impactos cruzados (Tabla 4): El objetivo de este método es hacer destacar los escenarios más probables, y también examinar las combinaciones de hipótesis que serán excluidas a priori. Si se considera un sistema de N hipótesis, el método SMIC, a partir de informaciones facilitadas por expertos, posibilita elegir entre varios escenarios de conformidad con su probabilidad de realización. (Deusto, s.f.)

El Método de Escenarios (Tabla 5): Un escenario, elemento clave de este método, es una descripción de las circunstancias, condiciones o acontecimientos que pueden representar la situación del entorno en un momento futuro del tiempo (T.H., 1968). Definido así, un escenario no es una previsión del futuro sino un análisis cualitativo de cómo puede ser dicho futuro (Vergara, 2010), y cómo se puede alcanzar. (Campos, 2010).

\section{¿Por qué la innovación?}

La innovación se considera un activo en las organizaciones (Luan., 2012), y se debe asignar como una función dentro de la estructura organizativa, ya que la habilidad para competir, crecer y desarrollarse depende de la capacidad de innovación que posean las organizaciones (Arraut, 2008).

De acuerdo a (Latín, Etimologías, 2016), la palabra "innovación" viene del latín innovatio y significa "acción y efecto de crear algo nuevo". Sus componentes léxicos son el prefijo "in" (penetración, estar en), novus (nuevo), más el sufijo -ción (acción y efecto). (Schumpeter J. , 1935), no ofrece una conceptualización ideal para la innovación, pero aporta que "la innovación consiste no sólo en nuevos productos y procesos, sino también en nuevas formas de organización, nuevos mercados y nuevas fuentes de materias primas" (Berry, 1994). Del mismo modo, (Rothwell., 1992), precisa la innovación como "un proceso que incluye la técnica, el diseño, la fabricación y las actividades comerciales y de gestión implicadas en la venta de un nuevo producto o el uso de un nuevo proceso de fabricación o equipamiento".

El Manual Frascati amplía la innovación hacia un concepto más técnico y más aplicado a la empresa "la transformación de una idea en un producto vendible, nuevo o mejorado, en un proceso operativo en una industria y en el comercio o un nuevo método de servicio social" (OECD, 2015). Estas transformaciones producen cambios dentro del ambiente 


\section{Revista científica, arbitrada e indizada, bajo la modalidad electrónica.}

organizacional que también están cubiertos bajo el concepto de "innovación” ya que según Suárez Mella (2009):

Cuando se convierten las ideas y los conocimientos en productos, procesos o servicios, entonces tiene su radical en una innovación. Es transformar el conocimiento y las ideas en riqueza. Por lo tanto, innovación no es añadir mayor sofisticación tecnológica a los productos, sino que estos se adapten mejor a las necesidades del mercado. Consiste en aportar algo nuevo y aún desconocido en un determinado contexto.

\section{Clasificación de la innovación:}

La innovación no solo abarca el componente tecnológico, sino el comercial y el organizativo, por lo que tiene presente las áreas siguientes: Introducción de un nuevo producto (Jiménez Jiménez, 2006); Introducción de un nuevo método de producción; Apertura de un nuevo mercado (Avendaño C., 2012). Descubrimiento de nuevos suministros de materias primas o de productos y Reorganización de una industria (Barreras, 2015).

Esto constata la idea de que la innovación va más allá de un invento. De conformidad con (Schumpeter J. , 1911), la innovación es la imposición de una novedad técnica u organizacional en el proceso de producción y no simplemente el correspondiente invento.

Es importante, pues, entender que la mejor forma de innovación es la que suma sostenibilidad a cada una de las áreas de nuestro lugar de trabajo (Odremán, 2014).

\section{Metodología:}

A fin de identificar las variables que participarían en el sistema se seleccionaron y entrevistaron a 13 expertos con altos cargos en empresas logísticas. Ellos ofrecieron un conjunto de ideas generales y específicas acerca de los elementos integrantes del sistema. Se llevó el siguiente procedimiento:

Depuración y clasificación de las ideas de acuerdo a temas compatibles.

Elaboración y aplicación de un cuestionario a una muestra de 14 empresas del sector para comprobar la validez y la fiabilidad de la consistencia interna de este instrumento.

Estimación del Alfa de Cronbach, cuyo resultado fue de .878, estimado como bueno para el fin designado y con ello se confirmó el uso de las variables antes mencionadas.

El análisis estructural se ocupa de relacionar las variables en un tablero de doble entrada o matriz de relaciones directas, por lo que se solicitó a los expertos la evaluación correspondiente (Ver tabla 1). Fue evaluada la dependencia y la motricidad entre variables. $\mathrm{Al}$ respecto, la ponderación que se utilizó fue la siguiente:

Influencia nula $=0$; Influencia directa débil o potencial $=1$; Influencia directa media (indirecta) $=2$; Influencia directa fuerte: $($ directo $)=3$.

La sumatoria de las filas de la variable indica las veces que cada una impactó en forma directa sobre el sistema. Al porcentaje y calificación en las dos últimas columnas se le denomina el índice de motricidad.

La sumatoria por columna representa las veces que cada una de las variables es influida directamente por las restantes y al porcentaje se le denomina índice de dependencia Una vez obtenidas las variables se continuó con la evaluación por parte de los expertos mediante el método Delphi, obteniendo los siguientes resultados: 


\section{Revista científica, arbitrada e indizada, bajo la modalidad electrónica.

Tabla 1.

Matriz de análisis estructural: (MICMAC)

\begin{tabular}{|c|c|c|c|c|c|c|c|c|c|c|c|c|c|c|c|c|c|c|c|c|c|c|c|c|c|c|c|}
\hline Variable & NOMBRE & $\mathrm{v}_{1}$ & $\mathrm{v}_{2}$ & $\mathrm{v}_{3}$ & $\mathrm{vu}$ & 15 & $\mathrm{~V} 6$ & $\mathrm{v} 7$ & v8 & 19 & vio & $\mathrm{vit}$ & Y12 & m3 & & V15 & 116 & vin & v18 & $v 19$ & 12012 & 1.12 & v2s & 124 & v25 & $\begin{array}{ll}\text { Motrida } \\
d\end{array}$ & $\%$ \\
\hline V1 & Fata de conocimiento del mercado $\mathrm{A} 1$ & & 2 & 0 & 0 & 0 & 1 & 1 & 1 & 0 & 0 & 0 & 0 & 0 & 1 & 0 & 0 & 0 & 0 & 0 & \begin{tabular}{l|l}
0 & 1
\end{tabular} & 1 & 0 & 1 & 1 & 10 & $3 \%$ \\
\hline $\mathrm{V} 2$ & $\begin{array}{l}\text { Inexistencia de activd estatágica para el éxito de la } \\
\text { innovación } A 7\end{array}$ & 2 & & 1 & 0 & 2 & 1 & 1 & 2 & 1 & 0 & 0 & 1 & 1 & 2 & 1 & 1 & 1 & 1 & 1 & \begin{tabular}{l|l}
0 & 0
\end{tabular} & 2 & 0 & 0 & 0 & 21 & $6 \%$ \\
\hline V 3 & Resistencia al cambio $\mathrm{A} 11,12$ & 2 & 2 & & 2 & 0 & 0 & 0 & 2 & 0 & 0 & 0 & 0 & 0 & 0 & 2 & 0 & 2 & 0 & 0 & \begin{tabular}{l|l}
0 & 0 \\
\end{tabular} & 2 & 0 & 0 & 0 & 14 & $4 \%$ \\
\hline 14 & Trabajo enequip o A9 & 0 & 1 & 0 & & 0 & 0 & 0 & 0 & 1 & 0 & 0 & 0 & 0 & 2 & 0 & 0 & 2 & 0 & 0 & \begin{tabular}{l|l}
0 & 0 \\
\end{tabular} & 0 & 0 & 0 & 0 & 6 & 2246 \\
\hline$\sqrt{5}$ & Fata de designacónde recursos f́nancieros B17 & 1 & 0 & 0 & 0 & & 0 & 0 & 1 & 0 & 0 & 2 & 2 & 0 & 3 & 0 & 0 & 0 & 1 & 0 & \begin{tabular}{l|l}
0 & 0 \\
\end{tabular} & 0 & 1 & 0 & 0 & 11 & $3 \%$ \\
\hline$v_{6}$ & Tiempo dedicado a innovar B18 & 0 & 0 & 0 & 0 & 1 & & 0 & 0 & 2 & 0 & 0 & 0 & 1 & 1 & 0 & 0 & 0 & 0 & 0 & \begin{tabular}{l|l}
0 & 0 \\
\end{tabular} & 0 & 0 & 0 & 0 & 5 & $1 \%$ \\
\hline V & Estuctura orgánica ade cuada B 20 & 0 & 0 & 0 & 0 & 0 & 0 & & 2 & 1 & 0 & 0 & 1 & 3 & 1 & 1 & 1 & 0 & 0 & 0 & \begin{tabular}{l|l}
0 & 0 \\
\end{tabular} & 0 & 1 & 0 & 0 & 11 & $3 \%$ \\
\hline VB & Capacitación del personal B21 & 1 & 1 & 1 & 2 & 0 & 1 & 1 & & 1 & 0 & 0 & 0 & 1 & 2 & 3 & 1 & 0 & 1 & 0 & \begin{tabular}{l|l}
0 & 0 \\
\end{tabular} & 0 & 0 & 0 & 0 & 16 & $5 \%$ \\
\hline V9 & Cono ámiento de la innovacóón B23 & 0 & 3 & 0 & 0 & 0 & 3 & 1 & 2 & & 0 & 0 & 2 & 1 & 2 & 1 & 1 & 0 & 1 & 1 & \begin{tabular}{l|l}
0 & 0 \\
\end{tabular} & 1 & 1 & 0 & 0 & 20 & $6 \%$ \\
\hline v10 & Pocade & 3 & 2 & 0 & 0 & 1 & 1 & 0 & 0 & 2 & & 1 & 0 & 1 & 0 & 0 & 0 & 1 & 1 & 1 & \begin{tabular}{l|l}
0 & 1 \\
\end{tabular} & 0 & 0 & 0 & 0 & 15 & 49 \\
\hline V11 & acóón B27 & 0 & 0 & 0 & 0 & 2 & 0 & 0 & 1 & 1 & 0 & & 0 & 0 & 0 & 1 & 0 & 0 & 0 & 1 & \begin{tabular}{l|l}
0 & 0
\end{tabular} & 1 & 0 & 0 & 2 & 9 & $3 \% 6$ \\
\hline V12 & Carenc & 0 & 1 & 0 & 0 & 1 & 1 & 1 & 1 & 1 & 1 & 1 & & 1 & 2 & 1 & 0 & 2 & 0 & 0 & $1 \mid 1$ & 0 & 1 & 0 & 1 & 18 & $5 \%$ \\
\hline v13 & Cultura de investgación de mercad os B32 & 2 & 2 & 0 & 0 & 2 & 1 & 1 & 2 & 1 & 2 & 0 & 0 & & 1 & 0 & 1 & 0 & 0 & 0 & \begin{tabular}{l|l}
1 & 1
\end{tabular} & 0 & 0 & 0 & 0 & 17 & $5 \%$ \\
\hline V14 & Personal dedicado a & 0 & 2 & 0 & 0 & 2 & 0 & 1 & 2 & 3 & 1 & 1 & 1 & 3 & & 0 & 2 & 0 & 2 & 2 & \begin{tabular}{l|l}
0 & 0
\end{tabular} & 1 & 0 & 0 & 0 & 23 & $7 \%$ \\
\hline V15 & Se imova con los RH existentes B39 & 0 & 2 & 0 & 2 & 2 & 1 & 1 & 1 & 2 & 0 & 0 & 2 & 2 & 3 & & 1 & 0 & 1 & 1 & \begin{tabular}{l|l}
0 & 0
\end{tabular} & 1 & 0 & 0 & 0 & 22 & $6 \%$ \\
\hline V16 & Cuenta con apoyo de Centos Educatios $B 42$ & 0 & 0 & 0 & 0 & 0 & 0 & 0 & 2 & 2 & 0 & 0 & 0 & 1 & 0 & 2 & & 0 & 1 & 1 & \begin{tabular}{l|l}
0 & 0
\end{tabular} & 2 & 0 & 0 & 2 & 13 & 446 \\
\hline V17 & Dif́cutad en la implementacoón de innovacón B38 & 2 & 1 & 0 & 0 & 1 & 2 & 1 & 1 & 1 & 0 & 0 & 1 & 2 & 1 & 0 & 0 & & 3 & 2 & 23 & 1 & 1 & 0 & 1 & 26 & $70 \%$ \\
\hline V18 & Apoyo de instuciones nacionales o interna B-44 & 2 & 0 & 0 & 0 & 0 & 2 & 0 & 2 & 1 & 0 & 2 & 0 & 1 & 3 & 0 & 0 & 1 & & 1 & \begin{tabular}{l|l}
0 & 0 \\
\end{tabular} & 0 & 1 & 0 & 0 & 16 & $5 \%$ \\
\hline V99 & $\begin{array}{l}\text { Poco access a los Centros de Investigación y } \\
\text { Desarollo D }\end{array}$ & 3 & 2 & 0 & 0 & 1 & 2 & 0 & 3 & 3 & 1 & 1 & 0 & 1 & 2 & 1 & 0 & 2 & 0 & & $0 \mid 0$ & 1 & 2 & 0 & 0 & 25 & \\
\hline v20 & Trámites burocáticos D50 & 0 & 0 & 0 & 0 & 0 & 0 & 0 & 0 & 0 & 0 & 1 & 1 & 0 & 0 & 0 & 0 & 0 & 0 & 0 & 3 & 3 & 2 & 0 & 2 & 12 & $3 \%$ \\
\hline V21 & Poca ayuda f́scal D51 & 0 & 0 & 0 & 0 & 0 & 0 & 0 & 0 & 0 & 0 & 3 & 0 & 0 & 0 & 0 & 0 & 0 & 0 & 0 & 0 & 0 & 0 & 0 & 3 & 6 & 234 \\
\hline V22 & Poca di wigación de programas de innovación D54 & 0 & 0 & 0 & 0 & 0 & 0 & 0 & 0 & 3 & 1 & 0 & 0 & 0 & 0 & 0 & 0 & 2 & 0 & 0 & \begin{tabular}{l|l}
0 & 0 \\
\end{tabular} & & 0 & 0 & 2 & 8 & 234 \\
\hline V23 & a el accoesso a la tecnología & 2 & 0 & 0 & 0 & 0 & 0 & 0 & 0 & 0 & 3 & 0 & 0 & 0 & 0 & 0 & 0 & 0 & 3 & 1 & 10 & 3 & & 3 & 0 & 16 & 59 \\
\hline V24 & Lacon & 0 & 0 & 0 & 0 & 0 & 0 & 0 & 0 & 0 & 0 & 0 & 0 & 0 & 0 & 0 & 0 & 0 & 0 & 0 & \begin{tabular}{l|l}
0 & 0 \\
\end{tabular} & 0 & 0 & & 0 & 0 & 02 \\
\hline \multirow[t]{3}{*}{ V25 } & Poca facitació & 0 & 0 & 0 & 0 & 0 & 0 & 0 & 0 & 0 & 0 & 0 & 0 & 0 & 0 & 0 & 0 & 3 & 3 & 2 & \begin{tabular}{l|l}
0 & 0 \\
\end{tabular} & 0 & 0 & 0 & & 8 & 2 \\
\hline & CIA & 20 & 21 & 2 & 6 & 15 & 16 & 9 & 25 & 26 & 9 & 12 & 11 & 19 & 26 & 13 & 8 & 16 & 18 & 14 & \begin{tabular}{l|l}
5 & 10 \\
\end{tabular} & $\begin{array}{ll}0 & 19 \\
\end{array}$ & 10 & 4 & 14 & 348 & $100 \%$ \\
\hline & & $6 \%$ & $6 \%$ & $1 \%$ & $2 \%$ & $4 \%$ & & $3 \%$ & $7 \%$ & $7 \%$ & & $3 \%$ & $3 \% 6$ & $5 \%$ & $7 \%$ & $4 \%$ & $2 \%$ & $5 \%$ : 5 & $5 \%$ & & $13 \%$ 3\%, & & $3 \%$ & $13 \%$ & $4 \%$ & $100 \%$ & \\
\hline
\end{tabular}

Fuente: Elaboración propia (2018)

\section{Resumen de dependencia por variable. (Eje $x$ ). (Tabla 1).}

Se aprecian las variables de acuerdo a su nivel de dependencia:

- Dependencia alta: V1, V2, V8, V9, V14, V15, V22.

Tienen alta dependencia de otras variables del sistema, lo cual significa que una variación, tendrán efecto en estas.

- Dependencia mediana: V5, V6, V10, V11, V12, V13, V16, V17, V18, V19, V21, V23, V25. Siguen la trayectoria del sistema, pero no inciden en sistema. Son necesarias y cualquier cambio en las otras variables las afectaría muy poco

- Dependencia baja: V3, V4, V7, V10, V20, V24. Son variables secundarias que no inciden en la evolución del sistema. Aportan, pero no son indispensables. 


\section{Revista científica, arbitrada e indizada, bajo la modalidad electrónica.}

\section{Resumen de motricidad por variable. (Eje y). (Tabla 1).}

Se puede apreciar las variables de acuerdo a su nivel de influencia (motricidad):

Motricidad alta: V2, V9, V15, V16, V17, V19.

Motricidad mediana: V1, V3, V5, V7, V8, V10, V13, V14, V18, V20, V23.

Motricidad baja: V4, V6, V11, V12, V21, V22, V24, V25.

A continuación, se colocan las variables en el plano cartesiano con el fin de elaborar un diagrama de dispersión: (Gráfica 1) y conocer las que más impactan al sistema (Zona de conflictos) y se deben tomar en consideración para el estudio:

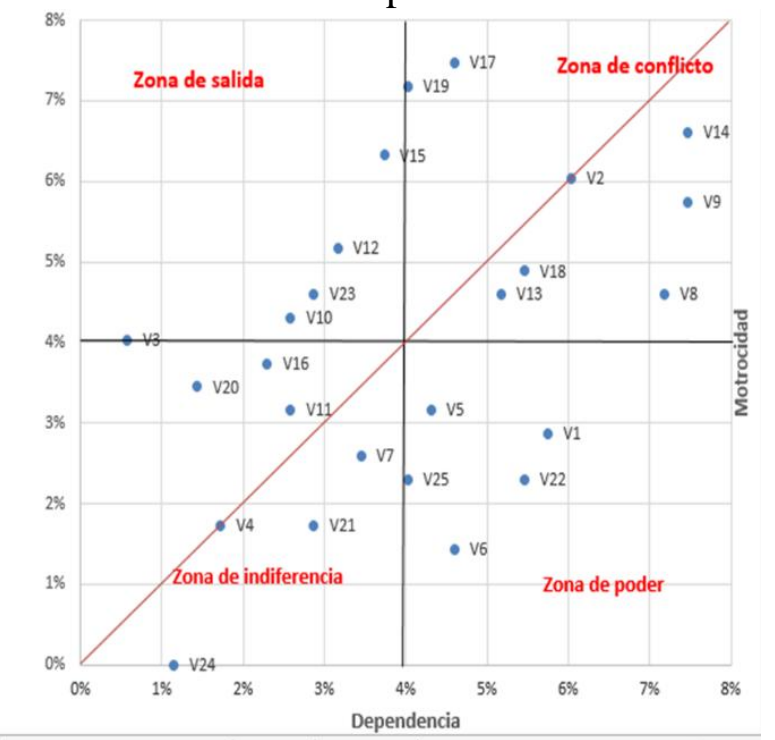

Gráfica 1: Diagrama de Dispersión

Fuente: Elaboración propia (2019)

En este esquema se ubica la posición de cada variable en los cuadrantes y su disposición con respecto a la diagonal. El eje vertical del gráfico representa la motricidad y el eje horizontal representa la dependencia.

\section{Resumen de variables por zonas}

En conjunto se observa 8 variables dispuestas en la zona de indiferencia. 5 variables en la zona de poder. 8 variables en la zona de conflictos y 4 variables en la zona de salida. Total: 25 variables (Tabla 2 ).

Con las variables que se sitúan en el área de conflicto se debe realizar el análisis, ya que son las que afectan todo el sistema de variables. A continuación, el resumen (Tabla 2) y la definición de actores (Tabla 3) que deben intervenir para permitir el desarrollo de la innovación en un futuro en las empresas logísticas y con ello logar un posicionamiento con las ventajas correspondientes. 


\section{Revista científica, arbitrada e indizada, bajo la modalidad electrónica.}

Tabla 2.

Resumen de variables por zonas

\begin{tabular}{|c|c|c|c|c|c|}
\hline & & \multicolumn{4}{|c|}{ Zonas del cuadrante } \\
\hline Varlable & Nombre & Inditerencla & Poder & Confilleto & Sallda \\
\hline v1 & Falta de conoclmlento del mercado A-1 & & $x$ & & \\
\hline V2 & Inexistencla de adtud estrateglca para el exito de la Innovacion A7 & & & $x$ & \\
\hline v3 & Resistencla al camblo A11, 12 & $x$ & & & \\
\hline V4 & Trabajo en equipo A9 & $x$ & & & \\
\hline v5 & Falta de designadon de recursos financleros B17 & & $x$ & & \\
\hline v6 & Tlempo dedicado a Innovar B18 & & $x$ & & \\
\hline v7 & Estructura organica adecuada 8 20 & $x$ & & & \\
\hline v8 & Capacitacion del personal 821 & & & $x$ & \\
\hline v9 & Conocimlento de la Innovadón $\overline{B 23}$ & & & $x$ & \\
\hline v10 & Poca demanda del producto Innovador 826 & & & & $x$ \\
\hline v11 & Altos costos de Innovacion 827 & $x$ & & & \\
\hline V12 & Carencla de un Centro de Innovaclon Logrstica & & & & $x$ \\
\hline v13 & Cultura de Investlgaclon de mercados B32 & & & $x$ & \\
\hline v14 & Personal dedicado a Innovacion y desarrollo B34 & & & $x$ & \\
\hline v15 & Se Innova con los RH existentes B39 & & & & $x$ \\
\hline V16 & Cuenta con apoyo de Centros Educativos B42 & $x$ & & & \\
\hline v17 & Dificultad en la Implementacion de Innovacion B38 & & & $x$ & \\
\hline v18 & Apoyo de Instituclones naclonales o Interna B-44 & & & $x$ & \\
\hline v19 & Poco acceso a los Centros de Investigacion y Des arrollo D & & & $x$ & \\
\hline v20 & Tramites burocraticos D50 & $x$ & & & \\
\hline V21 & Poca ayuda flscal D51 & $x$ & & & \\
\hline V22 & Poca divulgaclon de programas de Innovaclon D54 & & $x$ & & \\
\hline v23 & Impedimento para el accoeso a la tecnologra & & & & $x$ \\
\hline V24 & La competencla $A 3$ & $x$ & & & \\
\hline V25 & Poca fadiltaclon por parte del goblerno & & $x$ & & \\
\hline
\end{tabular}

Fuente: Elaboración propia (2019)

Se podrá apreciar que las diversas variables ubicadas en el área de conflictos tienen una relación fuerte, media, débil o potencial con la V12: Carencia de un Centro de Innovación Logística, la cual fue una de los principales elementos en el área de motricidad. Esta afecta a las demás variables. (Ilustración 1.)

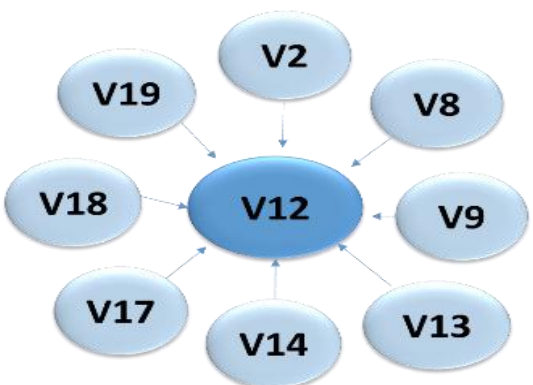

Ilustración 1: Relación de variables con V12

Fuente: Elaboración propia (2019) 


\section{Revista científica, arbitrada e indizada, bajo la modalidad electrónica.}

\section{Definición y funciones de actores}

Para definir los actores se llevó a cabo un análisis acerca de las instituciones que deben intervenir y qué funciones deben realizar para el desarrollo de la innovación en las empresas logísticas. A continuación, se puede observar los protagonistas resultantes. En este caso el Gobierno de Panamá como un ejemplo. (Tabla 3)

Tabla 3.

Definición de actores

\begin{tabular}{|c|c|c|}
\hline & NOMBRE & DESCRIPCIÓN \\
\hline A1 & Gobierno local & Gobierno de Panamá \\
\hline A2 & $\begin{array}{l}\text { Clientes de la empresa } \\
\text { logística }\end{array}$ & $\begin{array}{l}\text { Son aquellos usuarios de los productos o servicios, leales o desleales a la } \\
\text { empresa }\end{array}$ \\
\hline A3 & $\begin{array}{|lr|}\text { Centros } & \text { de } \\
\text { Investigación } & y \\
\text { Desarrollo } & \text { para } \\
\text { transferir tecnología }\end{array}$ & $\begin{array}{l}\text { Centros gubernamentales o privados en donde se realizan investigación y } \\
\text { desarrollo para adquirir nuevos conocimientos que ofrezcan productos } \\
\text { servicios innovadores }\end{array}$ \\
\hline A4 & $\begin{array}{l}\text { Universidades } \\
\text { Centros Educ ativos }\end{array}$ & $\begin{array}{l}\text { Son aquellos que ofrecen al personal especializado en el área que requiere la } \\
\text { empresa para su innovación }\end{array}$ \\
\hline A5 & Merc ado y competencia & Es el conjunto de personas que tienen un gran interés en adquirir los bienes 0 \\
\hline A6 & La empresa innovadora & $\begin{array}{l}\text { Se trata de la propia empresa cuya organización coincide con característic as } \\
\text { innovadoras }\end{array}$ \\
\hline A7 & $\begin{array}{l}\text { Centros de apoyo : } \\
\text { Senacyt, Asociaciones } \\
\text { gremiales de logística. } \\
\text { Apede }\end{array}$ & $\begin{array}{l}\text { es el esfuerzo de la empresa a traves de un Centro que le permita realizar } \\
\text { pruebas, ensayos y detalles de la innovación }\end{array}$ \\
\hline
\end{tabular}

Fuente: Elaboración propia (2019)

Una vez obtenida la información de la tabla 3, se siguió con una ponderación y evaluación de los actores o participantes de acuerdo a la importancia, influencia y dependencia de cada uno. Con los resultados se elaboró el diagrama de dispersión que a continuación se destaca: (Gráfica 2)

Se puede apreciar que en la zona de conflictos se ubican las variables (A3), (A6), (A7). En la zona de salida no se situó ninguna variable, en la zona de indiferencia se hallaron las variables (A2) y (A5). Y en la zona de poder las variables (A1) y (A4). (Gráfica 2). Por lo que se hace necesaria la participación de los Centros de Investigación y Desarrollo para Transferir Tecnología (A3), la empresa innovadora (A6) y Centros de apoyo como Senacyt para promover la innovación en empresas logísticas. 


\section{Revista científica, arbitrada e indizada, bajo la modalidad electrónica.}

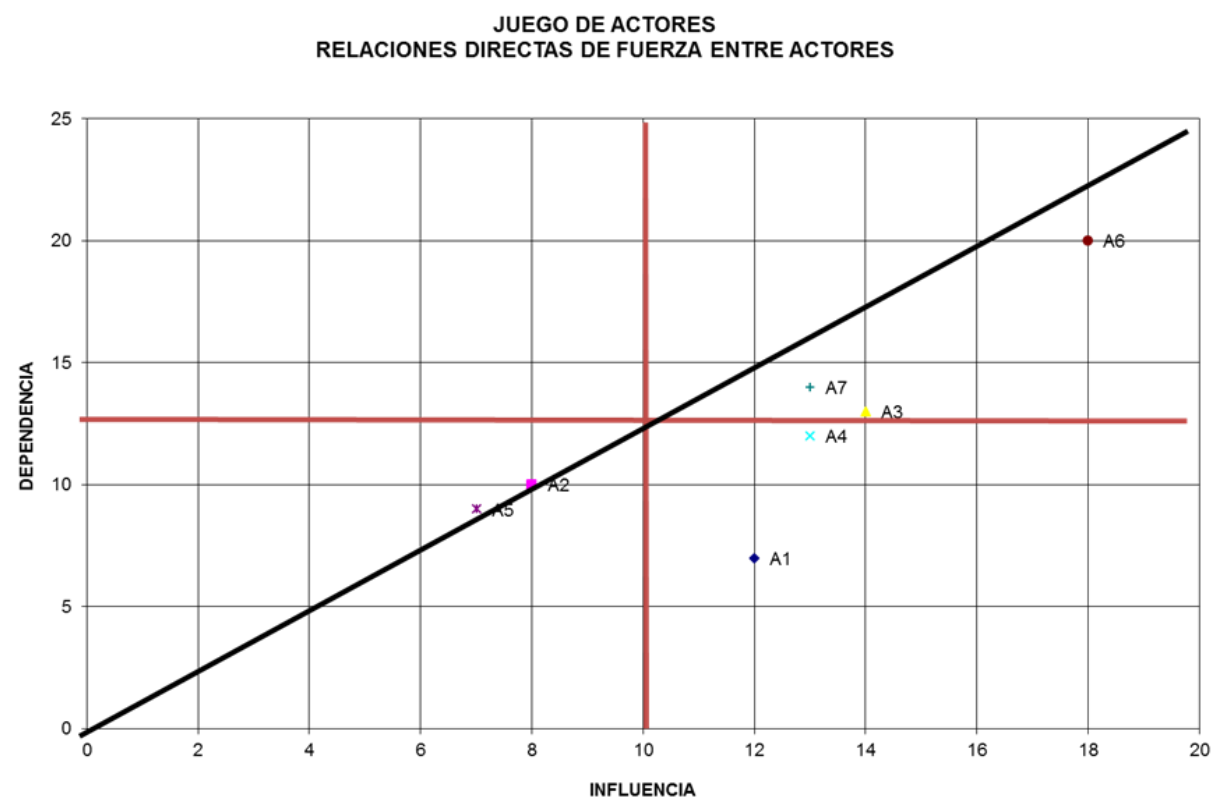

Gráfica 2. Diagrama de dispersión juego de actores

Fuente: Elaboración propia (2019)

Con la información detallada anteriormente se postularon 3 hipótesis futuristas y que se mencionan a continuación:

H 1: En los próximos dos años las empresas del sector logístico aumentarán el monitoreo de datos de mercado para conocer cambios en los comportamientos de los clientes y las relaciones de la competencia

H 2: En el período 2019 - 2022 las empresas logísticas promoverán la filosofía de gestión creativa e innovación

H 3: De tres a cinco años el sector logístico contará con un Centro de Investigación y

Desarrollo para la Innovación en la Cadena de Suministro

En la matriz de impactos cruzados se evalúan las probabilidades (Tabla 4) y En la matriz de escenarios se observan los resultados. (Tabla 5) 


\section{Revista científica, arbitrada e indizada, bajo la modalidad electrónica.}

Tabla 4.

Matriz de impactos cruzados MÉTODO DE ESCENARIO

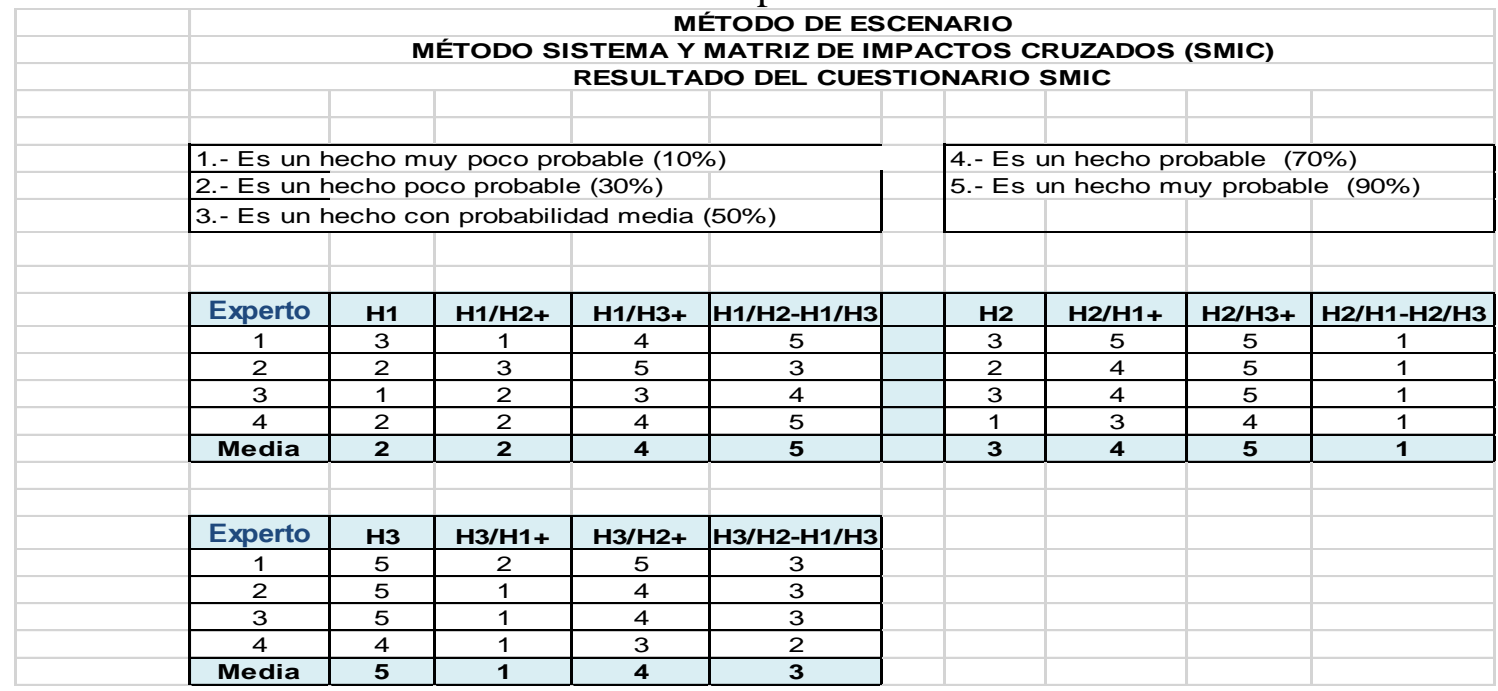

Fuente: Elaboración propia (2019)

La matriz de impactos cruzados permite observar las evaluaciones de los expertos y se toman en consideración los valores de acuerdo a la media para la elaboración de la siguiente matriz de escenario (Tabla 5)

Tabla 5.

Matriz de escenarios futuros

\begin{tabular}{|c|c|c|c|c|c|}
\hline HIPÓTESIS & OPTIMISTA & MODERADO & PESIMISTA & $\begin{array}{c}\text { PROBABILIDAD DE } \\
\text { OCURRENCIA } \\
\text { ABSOLUTA }\end{array}$ & $\begin{array}{l}\text { PROBABILIDAD DE } \\
\text { OCURRENCIA } \\
\text { CONDICIONADA }\end{array}$ \\
\hline H3 & $\begin{array}{llr}\text { Dentro de tres a cinco } \\
\text { años el sector logístico } \\
\text { contará con un Centro } \\
\text { de } & \text { Investigación } & \text { y } \\
\text { desarrollo } & \text { para } & \text { la } \\
\text { Innovación } & \text { en } & \text { la } \\
\text { Cadena de } & \text { Suministro }\end{array}$ & & & $90 \%$ & $\begin{array}{c}\text { Si se cumple: } \\
\text { H1, H2 === } 90 \% \text {. Si no } \\
\text { se cumple: H1 } \mathrm{H} 2 \\
===50 \%\end{array}$ \\
\hline $\mathrm{H} 2$ & & 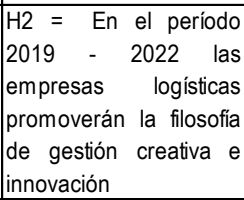 & & $50 \%$ & $\begin{array}{l}\text { Si se cumple: } \\
\mathrm{H} 1, \mathrm{H} 3===50 \% \text {. Si no } \\
\text { se cumple: H1, H3 } \\
===30 \%\end{array}$ \\
\hline $\mathrm{H} 1$ & & & 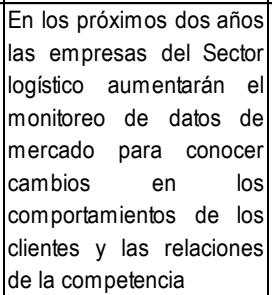 & $30 \%$ & $\begin{array}{c}\text { Si se cumple: } \\
\text { H2, H3 === } 30 \% . \quad \text { Si no } \\
\text { se cumple: H2, H3 } \\
===10 \%\end{array}$ \\
\hline
\end{tabular}

Fuente. Elaboración propia (2019) 


\section{Revista científica, arbitrada e indizada, bajo la modalidad electrónica.}

Las innovaciones penetran en el mercado tan rápido que crean un vacío en el desarrollo de la cadena de suministro, específicamente en el movimiento de grandes volúmenes de mercancías de un lugar a otro impactando los costos, tiempos de entrega, entre otros. En esa virtud, los procesos de globalización generan grandes desafíos y mayor complejidad en la gestión de la cadena de suministro. Contar con un Centro de innovación en logística podrá prevenir el complicado futuro de las empresas del sector, específicamente con la tecnología. Este Centro, no es más que es una instalación, tipo laboratorio, equipadas con tecnología, y personal especializado en los diferentes procesos de la cadena de suministro en donde se reunirá grupos de empresas junto con personal de universidades y apoyo del estado a fin de desarrollar innovaciones de todo tipo: tecnología, productos, servicios, procesos, etc. Inclusive se agrega la función de la recolección de datos de mercado para ser utilizados por las empresas.

También hay que considerar que el crecimiento de la población y por ende, el de la demanda de bienes y servicios, la empresa deba incrementar sus recursos, bienes y servicios hacer frente a una gran demanda.

Es necesario analizar exhaustivamente cada inversión que se realice en innovación, a fin de optimizar los costos y obtener ganancias, ya que estos recursos podrían quedar ociosos y reducirían el beneficio al producirse una demanda real $-\mathrm{y}$ unos ingresos- inferiores a lo previsto.

Una previsión optimista supone que la empresa pueda contar dentro de tres a cinco años con un Instituto de Innovación en Logística con una probabilidad del (90\%), pero sólo, si se cumplen las otras dos premisas: la promoción de la gestión creativa a partir del 2019 al 2020 (H2), y el monitoreo de datos de mercado para conocer cambios en los comportamientos de los clientes y las relaciones de competencia (H3).

Una previsión pesimista, en el otro extremo, impedirá la estructuración de este Centro imposibilitando a la empresa la obtención de competencias para hacer frente a una demanda, que inclusive puede ser superior a lo previsto, con el consecuente decremento en el nivel de servicios e incremento en los costes de los recursos. Todo ello conducirá a una pérdida de valor e imagen, disminución del mercado, con una posible desaparición de la empresa y, por ende, un número reducido de innovaciones que no impactarían al mercado poniendo en riesgo la estabilidad empresarial.

De no cumplirse las últimas dos premisas, la probabilidad de estructurar un centro se reduce a un $50 \%$, ya que se requiere previamente de un cambio de actitud en lo referente al liderazgo empresarial.

Esta actitud debe dirigirse hacia la implantación de nuevas tecnologías, desmantelar las barreras al cambio, detectar los nuevos modelos de negocios, gestión del talento, la formación y las tendencias que marcarán el futuro.

Por otro lado, en un $30 \%$ de probabilidad de cumplimiento, en los próximos dos años las empresas del sector logístico aumentarán el monitoreo de datos de mercado para conocer cambios en los comportamientos de los clientes y las relaciones de la competencia, sólo si se tiene el Centro y se examine el mercado en forma constante. En caso contrario tendrá una posibilidad del 10\%, lo cual significa la nulidad de esta acción. 


\section{Revista científica, arbitrada e indizada, bajo la modalidad electrónica.}

\section{Juego de actores}

En la matriz de juego de actores (Tabla 6) se pueden resumir los resultados finales de esta investigación y se explica por sí sola. Su descripción forma parte de las conclusiones.

Tabla 6

Matriz de juego de actores

Matriz del Juego de Actores

\begin{tabular}{|c|c|c|c|c|c|c|c|c|}
\hline \multirow{3}{*}{\multicolumn{2}{|c|}{$\begin{array}{c}\text { Variable clave } \\
\text { Disfunciones }\end{array}$}} & \multicolumn{5}{|c|}{ Juego de actores } & \multirow{2}{*}{\multicolumn{2}{|c|}{ Eventos }} \\
\hline & & \multicolumn{2}{|c|}{ Alianzas y conflictos } & \multicolumn{3}{|c|}{ Estrateqias } & & \\
\hline & & Demanda & Organismos & Proyectos & Anhelos & Temores & Necesidades & Potencial \\
\hline \multirow{3}{*}{ 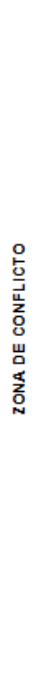 } & $\begin{array}{l}\text { Desconocimiento } \\
\text { del mercado }\end{array}$ & $\begin{array}{c}\text { Aumentar el monitoreo } \\
\text { de dat os de mercado } \\
\text { para conocer cambios } \\
\text { en los comportamientos } \\
\text { de los clientes y las } \\
\text { reacciones de la } \\
\text { competencia }\end{array}$ & $\begin{array}{c}\text { "Mercido y } \\
\text { competencis } \\
\text { Universidades } \\
\text { "Clientes de la } \\
\text { empresa }\end{array}$ & \begin{tabular}{|} 
Reforzar los vinculos \\
Universidgdes y \\
Centros Educativos, \\
los clientes para \\
realizar \\
investigaciones en \\
conjunto
\end{tabular} & $\begin{array}{c}\text { Información del } \\
\text { mercado access ible } \\
\text { con información } \\
\text { fresca scerca de } \\
\text { nuevas } \\
\text { necesidsdes de } \\
\text { productos y } \\
\text { sevicios } \\
\text { innovidores }\end{array}$ & \begin{tabular}{|c|} 
Información \\
disponible $a$ la \\
competencia
\end{tabular} & $\begin{array}{c}\text { Avanzar en un } 60 \% \\
\text { el levantamiento de } \\
\text { datos de mercado } \\
\text { para recabar } \\
\text { nueves } \\
\text { neces idades del } \\
\text { mercado que } \\
\text { cosdyuven a } \\
\text { producir innovación }\end{array}$ & $\begin{array}{l}\text { Existencia de } \\
\text { Centros de } \\
\text { Investigación de } \\
\text { mercados y/o } \\
\text { implementación } \\
\text { del área en la } \\
\text { empresa }\end{array}$ \\
\hline & $\begin{array}{c}\text { Inexistencia de } \\
\text { actitud estratégica } \\
\text { para el éxito de la } \\
\text { innovación }\end{array}$ & $\begin{array}{c}\text { Promover la filos ofía de } \\
\text { ges tión creativa e } \\
\text { innovación en la } \\
\text { empresa }\end{array}$ & $\begin{array}{l}\text { "La empresa. } \\
\text {-El gobierno. } \\
\text { "Centros de l\&D } \\
\text { "Centros de } \\
\text { apoyo }\end{array}$ & $\begin{array}{c}\text { Crear programas de } \\
\text { capacitación y } \\
\text { motivación, asi como } \\
\text { consultorias acerca } \\
\text { del cambio en la } \\
\text { cultura } \\
\text { ogranizacional para } \\
\text { orientarla a la nueva } \\
\text { gestión innovadora }\end{array}$ & $\begin{array}{c}\text { Cambio de actitud } \\
\text { hacia un modelo de } \\
\text { gestión del proceso } \\
\text { de innovación } \\
\text { empresarial }\end{array}$ & \begin{tabular}{|} 
No identificar \\
claramente los \\
factores claves \\
dentro de la \\
empres a que \\
caracteriz an \\
es te proceso
\end{tabular} & $\begin{array}{c}\text { Avanzar en un 50\% } \\
\text { en capacitación y } \\
\text { cambio cultural } \\
\text { hacia la innovación } \\
\text { dentro de la } \\
\text { empres a }\end{array}$ & $\begin{array}{c}\text { Dis ponibilidad } \\
\text { de } \\
\text { univers idades. } \\
\text { centros de } \\
\text { apoyo para } \\
\text { motiveción de } \\
\text { empressarios } \\
\text { pera innover }\end{array}$ \\
\hline & $\begin{array}{l}\text { Carencia de un } \\
\text { Centro de } \\
\text { Investigación y } \\
\text { Desarrollo para la } \\
\text { innovación en } \\
\text { cadena de } \\
\text { suministro }\end{array}$ & $\begin{array}{l}\text { Creación de un Centro } \\
\text { de Inves tigación y } \\
\text { Desarrollo para la } \\
\text { innovación en logística } \\
\text { y cadena de suminis tro }\end{array}$ & $\begin{array}{l}\text {-Gobierno } \\
\text { - Univers idides y } \\
\text { Centros } \\
\text { Educatios } \\
\text {-Centros de 18D } \\
\text { - Centros de } \\
\text { apoyo: Senacyt. } \\
\text { Asociaciones } \\
\text { gremiales en } \\
\text { logistica. Apede. }\end{array}$ & \begin{tabular}{|c|} 
Reforzar vinculos \\
entre instituciones \\
gubernamentales y la \\
empres $9 s$ de \\
logistica para la \\
oreación de un contro \\
de innovación \\
logístico
\end{tabular} & $\begin{array}{c}\text {-Aumentar la } \\
\text { productividad } \\
\text { empressrial } \\
\text { mediante la } \\
\text { inserción de nuevos } \\
\text { productos, senvicios } \\
\text { o métodos }\end{array}$ & $\begin{array}{c}\text { Que s ólo parte } \\
\text { del sector } \\
\text { responds a } \\
\text { esta propues ta }\end{array}$ & $\begin{array}{c}\text { Creación de un (1) } \\
\text { centro de l\&D en } \\
\text { el periodo } 2019 \text { - } \\
2022\end{array}$ & $\begin{array}{c}\text { Existencia de } \\
\text { todos los } \\
\text { estamentos } \\
\text { neces srios para } \\
\text { la formación de } \\
\text { es te centro }\end{array}$ \\
\hline
\end{tabular}

Fuente. Elaboración propia (2019)

La Creación del Centro de Innovación depende del conocimiento de las necesidades del mercado, el cual requiere de tres elementos para su adecuada estructuración: análisis del entorno, comportamiento de los consumidores y la competencia, mismo que será parte de un estudio posterior.

Algunos elementos a considerar para su consecución exitosa: personal capacitado, objetivos bien trazados, recursos financieros y la constante exploración del mercado. Una de las tendencias de este mercado y que, la cadena de suministros debe estar a la expectativa, es la micro segmentación, para identificar la categoría exacta de compra del consumidor. Para conocer esas categorías se requiere tener conocimiento profundo de esos segmentos de mercados o clientes individuales.

Estas tendencias se realizan con base a estudios de comportamientos de consumidores y usuarios en comparación con los datos históricos de la empresa. Con esta información de mercado se pueden desarrollar productos y servicios innovadores para planear y ejecutar estrategias rentables para los negocios.

Normalmente cuando se realizan monitoreo del mercado para conocer cambios en los clientes, las reacciones de la competencia y estudios de comercialización de productos o servicios en empresas logísticas, se refieren a aspectos cuantitativos con estudios que sólo toman en consideración las cantidades de productos para estimar la demanda futura, e

Revista Global Negotium

ISSN $2642-4800$

Vol. 2 No. 3 


\section{Revista científica, arbitrada e indizada, bajo la modalidad electrónica.}

ignoran las características cualitativas observables para la introducción de novedades, establecer los criterios de calidad y de personificación de la marca.

Cualquier buena idea perdería sus beneficios si la empresa no promueve la filosofía de gestión creativa e innovación dentro de su estructura orgánica

La demanda y sus variaciones periódicas son diferentes en cada mercado, la clave está en saber separar los hechos de la ficción, conocer las necesidades reales del negocio y las expectativas del consumidor para aproximarse a la innovación de la mano de la lógica, con el fin de emplazar el equilibrio entre deseo y realidad. Para ello la empresa debe rediseñar su estructura orgánica, aplicando recursos frescos para cumplir con las metas.

\section{CONCLUSIÓN}

De conformidad con lo mostrado, es preocupante ubicar la competencia entre empresas logísticas limitadas por la capacidad de respuesta y al acotamiento de costos. No obstante, el escenario antes descrito demanda una nueva visión, acorde con el entorno externo de la empresa, en el cual las estrategias deberán redefinirse de conformidad con las nuevas tendencias y colocar en un futuro próximo a las empresas desarrollando proyectos innovadores impactantes para la población. Para ello, tanto los actores ya descritos como los especialistas en el área deben trabajar en las siguientes variables que se desprenden de esta investigación:

\section{a) Desconocimiento del mercado}

Es una de las disfunciones encontradas en el sistema. La empresa desconoce los elementos del ambiente externo, por lo tanto, no puede obtener los beneficios, que este le puede ofrecer. Desconocen las acciones de la competencia que les puede afectar en un futuro próximo, por lo que es posible se estén perdiendo oportunidades valiosas.

Todos los productos o servicios son perfectibles y por eso la innovación debe estar presente en toda industria. La anticipación estratégica es vital para la permanencia en los mercados, pero es imprescindible el monitoreo de datos de mercado para conocer los cambios en los comportamientos de los clientes

Para ello es importante el Centro de Innovación en Logística que coadyuve a generar datos de mercados para la generación de novedades y que coadyuve a la empresa a gestar las innovaciones. Esta función se puede coordinar con el área de mercadeo de la empresa.

Las instituciones que deben intervenir son: instituciones de mercadeo, Universidades para los especialistas y los clientes de la empresa para conocer mayor información de su comportamiento.

Los temores más comunes es que la información que se obtiene del mercado también es compartida con la competencia, por lo que debe haber algún acuerdo para salvaguardar los secretos y propiedad intelectual hasta su registro.

Para que se pueda empezar a planear las diferentes innovaciones se debe avanzar en el levantamiento de la información por lo menos un 60\%. Hay que tener cuidado de que el objetivo y el instrumento esté dirigido hacia productos, servicios o procesos innovadores. 


\section{Revista científica, arbitrada e indizada, bajo la modalidad electrónica.}

\section{b) Inexistencia de una actitud estratégica para el éxito de la innovación:}

Es una de las causas por las cuales no se produce la innovación en las empresas logísticas, por ello es importante un cambio en esa actitud, mediante la promoción creativa innovadora y por otro lado, creando programas de capacitación y motivación, así como consultorías acerca del cambio en la cultura organizacional para orientarla a la nueva gestión innovadora.

Uno de los temores es no estar en la posibilidad de identificar claramente los factores claves dentro de la empresa que caracterizan este proceso, por lo que la oportunidad de hacer un cambio efectivo en la mentalidad organizativa de la empresa no permite la adecuación de la estructura orgánica.

Es recomendable que se avance por lo menos en un $50 \%$ en capacitación dirigido al cambio de cultura tradicional hacia la innovación como centro de la empresa. Este $50 \%$ significa por los menos los cursos y talleres básicos.

Para ello, se puede apoyar en universidades, tanto estatales como privadas y en centros de Coaching para la innovación.

c) Carencia de un Centro de Investigación y Desarrollo para la Innovación en

\section{Cadena de Suministro.}

Para su instalación se requiere del apoyo del gobierno, universidades, Centros educativos, Centros de I\&D y de Centros de Apoyo como Senacyt, Asociaciones Gremiales en logística, Apede.

Uno de los proyectos propuestos es del de reforzar vínculos entre instituciones gubernamentales y las empresas logísticas para la creación del Centro de Innovación Logística.

Con el uso de este Centro la empresa se podrá beneficiar mediante el incremento de la productividad empresarial mediante la inserción de nuevos productos, servicios o métodos. El temor es que sólo parte del sector responda a esta propuesta y no se obtenga los apoyos necesarios para su realización.

\section{REFERENCIAS BIBLIOGRÁFICAS}

Ancelin, C. (1983.). L'analyse structurelle : le cas du Vidéotex, Futuribles. París.

Arraut, L. C. (2008). La Innovación de Tipo Organizacional en las Empresas Manufactureras en Cartajena de Indias. Semestre Económico, 11(22), pp. 185 - 203. $\begin{array}{llllll}\text { Recuperado el } 04 \text { de } & \text { Octubre }\end{array}$ http://www.scielo.org.co/pdf/seec/v11n22/v11n22a9.pdf

Avendaño C., W. (2012). Innovación: Un proceso necesario para las pequeñas y medianas empresas del Municipio de San José Cúcuta. Redalyc, 15(31). doi:https://www.redalyc.org/pdf/1650/165024299008.pdf

Baena, P. G. (2005). Construcción del pensamiento prospectivo. México: Trillas.

Barreras, I. e. (2015). La innovación, competitividad y desarrollo tecnológico en las MIP y ME's del municipio de Angostura, Sinaloa*. Revista mexicana de ciencias agrícolas, 6(3). Recuperado el 04 de Octubre de 2019, de 


\section{Revista científica, arbitrada e indizada, bajo la modalidad electrónica.}

http://www.scielo.org.mx/scielo.php?script=sci_arttext\&pid=S200709342015000300013

Berry, M. \&. (1994). Managing Technology and Innovation: a review. $R \& D$ management, 24(4), $341-353$.

Campos, V. (2010). Wolters Kluver. Recuperado el 12 de Diciembre de 2018, de Método de los escenarios: http://diccionarioempresarial.wolterskluwer.es/Content/Documento.aspx?params=H 4sIAAAAAAAEAMtMSbF1jTAAASMTMwsjtbLUouLM_DxbIwMDS0NDQ3O QQGZapUt-ckhlQaptWmJOcSoAnNKyMDUAAAA=WKE

Chitu, O., \& Suzanne, P. (2004). The Delphi method as a research tool: an example, design considerations and applications. Elsevier, 42(1), $15 \quad-\quad 29$. doi:https://doi.org/10.1016/j.im.2003.11.002

Conceptodefinición.com. (s.f.). Definición del Método Delphi. Recuperado el 12 de Diciembre de 2018, de https://conceptodefinicion.de/metodo-delphi/

Deusto, U. (s.f.). Métodos de escenario. Recuperado el 2018 de Octubre de 04, de http://www.prospectiva.eu/zaharra/06_Smic_ESTE.pdf

Estrada Ruiz, M. (2014). La prospectiva de la participación social en la educación en México: el punto de vista de los especialistas. Scielo, 19(58). doi:http://dx.doi.org/10.1590/S1413-24782014000800011

Forrester, J. (1961). Industrial Dynamics. E.E.U.U.

Godet, M. \&. (2011). Strategic Foresight for Corporate and Regional Development. París: DUNOD - UNESCO - Fondation Prospective et Innovation.

Godet, M. (2000). La caja de las herramientas de la prospectiva estratégica. France: Gerpa.

Godet, M., \& Durance, P. (2011). Strategic Foresight for Corporate and Regional Development. Dunot Unesco.

Guzmán, A. e. (2005). Análisis Estructural: Técnica de la Prospectiva. París.

Inayatullah, S. (2012). "Estudios del futuro: teorías y metodologías", en Hay futuro. Visiones para un mundo mejor, . Madrid, BBVA,. Recuperado el 07 de Julio de 2019, de https://www.bbvaopenmind.com/articulos/estudios-del-futuro-teoriasmetodologias/

Inayutallah, S. (1990). Deconstructing and Reconstructing the Future. Futures 22, 22(2), 115 - 141. Recuperado el 27 de Junio de 2019

Instituto de Prospectiva Estratègica, IPE. (1995). Instituto de Propectiva Estratègica. Recuperado el 18 de Febrero de 2018, de http://www.prospecti.es/ipeframe.htm

Jiménez Jiménez, D. y. (2006). Innovación, aprendizaje organizativo y resultados empresariales. Un estudio empírico. Cuadernosde economía y dirección de la empresa, 29, 31 - 55. Recuperado el 04 de Octubre de 2019

Latín, Etimologías. (2016). Etimologías Chile. Chile: Dechile. Obtenido de http://etimologias.dechile.net/?innovacio.n

Luan., S. S. (2012). Management Innovation in Brazilian Technology Companies: The Challenges Faced by Managers in the Practice of Innovation. American Journal of Industrial and Business Management, 2(4). Recuperado el 04 de Octubre de 2019, 


\section{Revista científica, arbitrada e indizada, bajo la modalidad electrónica.}

de

http://www.scirp.org/(S(351jmbntvnsjt1aadkposzje))/journal/paperinformation.aspx ?paperid $=24052$

Miklos, T. (1995). Planeación prospectiva. México: Limusa.

Moctezuma, G., \& Tapia, A. e. (Julio/agosto de 2012). Estudio prospectivo medioambiental con orientación estratégica de la investigación forestal en seis países latinoamericanos. Scielo, 3(12). Recuperado el 03 de Octubre de 2019, de http://www.scielo.org.mx/scielo.php?script=sci_arttext\&pid=S200711322012000400002

Mojica, F. (1991). La prospectiva. Técnicas para visualizar el futuro. Colombia: Legis.

Montañana, A. (2004). Conocimiento prospectivo. México: Ciecas/Ipn.

Odremán, J. (2014). Gestión Tecnológica: Estrategias de Innovación y Transferencia de Tecnología en la Industria. Universidad, Ciencia y Tecnología, 18(73). Recuperado el 04 de $\quad$ Octubre $\quad$ de 2019, de http://ve.scielo.org/scielo.php?script=sci_arttext\&pid=S1316-48212014000400004

OECD. (2015). Fascati Manual. Paris: OECD.

Pinto, J. P. (2008). Las herramientas de la prospectiva estratégica: usos, abusos y limitaciones. Cuadernos de Administración. Recuperado el 27 de Junio de 2019, de $<$ http://www.redalyc.org/articulo.oa?id=225014905005>

prospective, L. (s.f.). Método de prospectiva. Recuperado el 11 de Diciembre de 2018, de http://es.laprospective.fr/Metodos-de-prospectiva/Los-programas/67-Micmac.html

Rothwell., R. (1992). Successful Industrial Innovation Critical Factors for the 1990s. $R \& D$ Management, 22(3), pp. 221-239. Recuperado el 04 de Octubre de 2019, de http://www.scirp.org/(S(351jmbntvnsjt1aadkposzje))/reference/ReferencesPapers.as px?ReferenceID $=607655$

Saint Paul R., T.-B. P. (1974). Innovation et évaluation technologiques : sélection des projets, méthodes de prévision. Francia: Entreprise Moderne d'Edition.

Schumpeter, J. (1911). Teoría del Desarrollo Económico. New York.

Schumpeter, J. (1935). Análisis del Cambio Económico. En Ensayos del ciclo económico. México: Fondo de Cultura Económica.

T.H., G. (1968). Initial experiments with the Cross-Impact Matrix. Futures, 1(2), 110 - 166. Recuperado el 2019 de Octubre de 04

Vergara, J. C. (2010). La planeación por escenarios. Propectiva, 8(2). Recuperado el 12 de Diciembre de 2018, de file://C:/Users/ydcantu/Downloads/DialnetLaPlaneacionPorEscenariosRevisionDeConceptos YPropu-3634575.pdf 